\title{
Tulsi-The matchless medicinal herb.
}

\author{
Dr.Vikram Perakath ${ }^{1}$ BDS, Dr. Reema Lakshmanan. ${ }^{2}$ M.D.S. \\ I(BDS ,Saveetha Dental College,Saveetha University,India) \\ 2(Department of Periodontics, Saveetha Dental College,Saveetha University,India)
}

\begin{abstract}
For many thousands of years,medicinal plants have been used to cure diseases, especially in the Indian subcontinent.Tulsi(Ocimum sanctum) is one such plant with many applications in medicine,like in periodontitis it influences cytokine production and has an immunomodulatory effect.Tulsi extracts are used as oral rinses.A proper understanding of its pharmacological profile is necessary to understand its various possible applications in medicine, and to pave the way for future studies in its applications in medicine.
\end{abstract}

Keywords:Tulsi(Ocimum sanctum),Periodontitis,Gingivitis,Cytokines,Neem(Azadirachta indica)

\section{Intoduction:}

Plants are the primary source of medicine. Medicinal plants are considered to be very rich sources of secondary metabolites and oils which are of therapeutic importance. The important advantages of medicinal plants in various treatments are: their safety besides being less expensive and easily availabile around the world [1]. Use of plants as a source of medicinal value is a very old concept. Chinese were the first to use plants as therapeutics before 4000- 5000 B.C. In India use of plants as a medicine appeared in Rigveda which was written around 3500 - 1600 B.C. Properties of plants as a source of medicine were studied in detail in Ayurveda which is considered the foundation of all the medical sciences.[2]

In the indian subcontinent Tulsi is considered as the most sacred plant and is often referred as the "Holy Basil". There is documented evidence of the use of Ocimum sanctum ( Tulsi) as an aromatic plant in Ayuveda. Belonging to the family Labiateae, It grows in tropical and sub tropical belts including India[3] and is commonly seen in the indian fields. "Tulsi" in Sanskrit means "the incomparable one"[4] and hence is considered the queen of herbs. In India two forms of Tulsi are common - dark or Shyama (Krishna) Tulsi and light or Rama Tulsi. The former possesses greater medicinal value and is commonly used for worship. Other species in India include O. canum, O. basilicum, O. kilimandscharicum, O. ammericanum, O. camphora and O. micranthum.[5][6]

This plant has been evaluated pharmacologically for antimicrobial, immunomodulatory, anti-inflammatory, hypoglycemic,chemoprotective and analgesic activities.[7]

An essential oil,prepared from its leaves, contains eugenol, eugenal, carvacrol, methylchavicol, limatrol and caryophylline. The leaves also contain ursolic acid and $\mathrm{n}$-triacontanol.The oil prepared from its seeds is composed of fatty acids and sitosterol. The roots contain sitosterol and three triterpenes A, B, and C.Eugenol, its methyl ether, nerol, caryophyllene, terpinen 4-decylaldehyde, selinene, pinenes, camphene and a-pinene have been identified in essential oil,it also contains rosmarinic acid, thymol, linalool and methyl chavicol and citral etc.[8].

Periodontitis is defined as an inflammation of the periodontium caused by a complex reaction initiated when subgingival polymicrobial plaque bacteria are in close contact with the epithelium of the gingival sulcus. Tissue destruction arises from toxins and enzymes produced by the bacteria and from host-mediated defense responses. Apical movement of the junctional epithelium, which indicates attachment loss and alveolar bone loss, is diagnostic of periodontitis.[9]

Periodontal disease is caused by plaque, which is formed from harmful bacteria. The mouth is full of bacteria which are harmless in variety. Periodontal disease usually develops because of an increase in bacteria quantity in the oral cavity and a change in balance of bacterial types from harmless to disease-causing bacteria. These harmful bacteria increase in mass and thickness until they form a sticky film called plaque.

In healthy mouths, plaque actually provides some barrier against outside bacterial invasion. When it accumulates to excessive levels, however, bacterial plaque sticks to the surfaces of the teeth and adjacent gums and causes infection with subsequent swelling, redness, and warmth.

When plaque is allowed to remain in the periodontal area, it transforms into calculus (commonly known as tartar).

New technologies have provided insights into how dental plaque functions as a biofilm. Confocal microscopy has confirmed that plaque has an open architecture similar to other biofilms, with channels and voids. Gradients develop in areas of dense biomass over short distances in key parameters that influence microbial growth and distribution. Bacteria exhibit an altered pattern of gene expression either as a direct result of being on a surface or indirectly as a response to the local environmental heterogeneity within the biofilm. 
Bacteria communicate via small diffusible signalling molecules (e.g. competence-stimulating peptide, CSP; autoinducer 2); CSP induces both genetic competence and acid tolerance in recipient sessile cells. Thus, rates of gene transfer increase in biofilm communities, and this is one of several mechanisms (others include: diffusionreaction, neutralization/inactivation, slow growth rates, novel phenotype) that contribute to the increased antimicrobial resistance exhibited by bacteria in biofilms. Oral bacteria in plaque do not exist as independent entities but function as a co-ordinated, spatially organized and fully metabolically integrated microbial community, the properties of which are greater than the sum of the component species.[10]

In the oral cavity,the bacteria exist in different niches.A niche is "The set of biotic and abiotic conditions in which a species is able to persist and maintain stable population sizes."

The most common niches in the oral cavity are Subgingival flora,Tongue coatings[11], Tonsillar fossae,Floor of the mouth.

The microflora in the mouth,can be classified as endogenous microflora and exogenous microflora.Endogenous microflora is microflora already present in the body, but has previously been inapparent or dormant.Exogenous bacteria are microorganisms introduced to closed biological systems from the external world.[12]

\section{Symptoms of periodontal disease typically progress over time and include:}

\subsection{Red and Swollen Gums,}

$1.2 \mathrm{Gum}$ Bleeding. Bleeding of the gums, even during brushing, is a sign of inflammation and the major marker of periodontal disease,

1.3Bad Breath.(Halitosis) Debris and bacteria can cause a bad taste in the mouth and persistent bad breath.There is an extensive list of possible causes of halitosis in the mouth alone, however by far the most prevalent causes reported are halitogenic biofilm on the posterior dorsal tongue and within gingival crevices and periodontal pockets (i.e. bacteria living on the back of the tongue and below the gumline, and in the pockets created by gum disease between teeth and the gums). Both of these main causes share a common theme of proteolytic putrefaction of sulfur containing amino acids in dietary and salivary protein by mostly anaerobic, Gram negative bacterial species.

The most common location for mouth related halitosis is the tongue.Tongue bacteria produce malodorous compounds and fatty acids, and account for 80 to $90 \%$ of all cases of mouth-related bad breath.Large quantities of naturally-occurring bacteria are often found on the posterior dorsum of the tongue, where they are relatively undisturbed by normal activity. This part of the tongue is relatively dry and poorly cleansed, and the convoluted microbial structure of the tongue dorsum provides an ideal habitat for anaerobic bacteria, which flourish under a continually-forming tongue coating of food debris, dead epithelial cells, postnasal drip and overlying bacteria, living and dead. When left on the tongue, the anaerobic respiration of such bacteria can yield either the putrescent smell of indole, skatole, polyamines, or the "rotten egg" smell of volatile sulfur compounds (VSCs) such as hydrogen sulfide, methyl mercaptan, Allyl methyl sulfide, and dimethyl sulfide.[13]

1.4Gum Recession and Loose Teeth. As the disease advances, the gums recede, and the supporting structure of bone is lost. Teeth loosen, sometimes causing a change in the way the upper and lower teeth fit together when biting down or how partial dentures fit.

1.5Abscesses. Deepening periodontal pockets between the gums and bone can become blocked by tartar or food particles. Infection-fighting white blood cells become trapped and die. Pus forms, and an abscess develops. Abscesses can destroy both gum and tooth tissue, cause nearby teeth to become loose and painful, and may cause fever and swollen lymph nodes.[14]

The progression of the disease Periodontitis results in the destruction of the periodontium,i.e,the alveolar bone and periodontal ligaments is brought about by the influence of Cytokines.Cytokines generally function as intra-cellular messenger molecules that evoke particular biological activities after binding to a receptor on a responsive target cell.The main biological activities include cellular and humoural responses,induction of inflammatory response,regulation of haematopoesis and induction of wound healing.Cytokine receptors,example,IL1,IL6,IL8,IFNalfa,IFNbeta,TNFalfa,TNFbeta,MIP-1[15]

Occimum sanctum extract reduces inflammation by inhibiting the release of pro inflammatory cytokines(like TNF \& IL1) and mediators(Nitric oxide).Specific cytokines help initiate and regulate various inflammatory processes.Nitric oxide, a chemical involved in various cell signalling reactions helps in the inflammatory cascade.Thus blocking the action of these two substances means blocking communication of inflammatory processes.

In 2011,Mondal S et.al. conducted a double blinded randomized control trial to study the 
immunomodultory effects of Ocimum sanctum.In the study,300mg capsules of alchoholic extracts of Ocimum sanctum were given to one group, and a placebo was given to another group,on an empty stomach.A total of 24 volunteers were used.The levels of Interferon gamma,Interleukin 4 and T-helper cells were checked after a period of four weeks. The study showed significantly icreased levels of these markers in the study group as compared to the control group.Thus it was shown that Ocimum sanctum has pro-immunomodulatory effects on the body.

Another study performed by Saina et.al in 2009, was performed on lab mice to study the effect of Ocimum sanctum oil and Clove(Syzgium aromaticum) on the induction of resistance to respiratory tract infection by klebsialla pneumonia.The study was performed in two regimens.one short term for 15 days and one long term for 30 days,during which Tulsi oil and clove oil was fed to lab mice infected with Klebsiella Pneumonia.The results showed a significant decrease in the bacterial colonization after short term feeding with clove oil and a significant decrease in bacterial colonization after long term feeding with tulsi.Thus it can be stated that Tulsi oil as a dietary supplement is effective against bacterial colonization of the lungs.

\author{
III. Prevention of progress of the disease,Periodontitis,can be brought about by:[16] \\ 3.1Toothbrushing, \\ 2.2Flossing, \\ 2.3Use of Toothpastes and Mouthwashes, \\ 2.4Change of lifestyle(like healthy eating and quitting smoking)
}

IV. Mouthwashes:

The following herbal mouthwashes have shown properties which may inculcate them in the treatment of periodontitis:

\title{
4.1Tulsi(Ocimum sanctum):
}

Tulsi is known as "Queen of plants" "The mother medicine of nature". Tulsi i.e. Ocimum sanctum is a plant with enormous properties for curing and preventing diseases. It is regarded as deity in Indian subcontinent. The genus Ocimum sanctum Linn. (Labiateae or Lamiaceae) comprises 30 species which are found in tropical and subtropical regions. Leaves and flowering tops are used for extracting essential oil. Oil of O. sanctum has revealed the presence of five fatty acids (stearic, palmitic, oleic, linoleic and linolenic acids). It is a good source of beta carotene, calcium, vitamin $\mathrm{C}$ and it also contains volatile substances (including estragol, linalool, eugenol, methyl chavicol and small quantities of methyl cinnamate, cineole, and other terpenes), tannins, camphor, flavonoids, triterpene: urolic acid. Leaves are diaphoretic, anti-periodic; they are also used in bronchitis, gastric and hepatic disorders. Decoction of leaves is recommended for cough, malaise and in colds. It is a good mosquito repellant as well. Oil extracted from flowers is used in skin diseases and ring worm infection.

Chewing of tulsi leaves helps clear ulcers and infections of the mouth.As a mouthwash it is useful against bad breath and for maintaining healthy gums.

A research conducted by Agarwal P.et.al showed that Tulsi extract demonstrated an antimicrobial property against streptococcus mutans.

Pharmacological profile of tulsi:

The essential oil present in most of the Ocimum species is responsible for its antifungal,antibacterial and anti viral properties.[17]The ethanolic extract of Ocimum sanctum was found to be effective against streptococcus mutans in an invitro study.Its leaves have been found to have a strong anti-fungal activity against the Aspergillus species.[18].Due to the fact that microorganisms develop resistance to available synthetic antibiotics,phytocemicals from various Ocimum species can be used as therapeutics.

Ocimum sanctum seed oil appears to modulate both humoral and cell-mediated immune responsiveness and these immunomodulatory effects maybe mediated by GABAergic pathways.A study showed increase in antibody production due to the release of mediators of hypersensitivity reactions and tissue responses in the target organs by Ocimum sanctum.

Ocimum sanctum has been shown to inhibit acute as well as chronic inflammation.The essential oil and seed extract act by the inhibition of cyclo-oxygenase and lipoxygenase.[19].

Ocimum sanctum maybe considered as a drug of natural origin which posseses both anti-inflammatory and antiulcer activity.It exhibits gastric secretion inhibition activity in pylorus ligated rats due to its lipooxygenase inhibitory,antihistaminic and antisecretory action.[20]

The use of Ocimum sanctum in the therapeutic management of diabetes mellitus is gaining a lot of importance.The rich fibre content of plant particularly the soluble fibres have shown beneficial effects in treating hyperglycemia and hyperlipidemia.The mechanism of action for its anti diabetic effect is that the extract of plant is able to stimulate adenylate cyclase or phosphatidylinositol,or having direct effect on pancreatic beta cells by promoting calcium entry and thereby causing release of stored insulin.[21] 
Ocimum sanctum has been found to reduce serum alanine aminotransferase( ALT) levels, and thus show a hepatoprotective activity.[22]

Aqueous and ethanolic extracts of leaves of Ocimum sanctum on oral administration and topical application of leaf paste reported to have significantly delayed the incidence of papillomas and carcinomas and icreased the survival rate. The mechanism behind it maybe that it enhances the mixed function oxidase system(MFO) and increased glutathione and glutathione $\mathrm{S}$ transferase enzymes,suggesting that the extracts may play a role in the detoxification of the carcinogens.[23]

\subsection{Neem(Azadirachta indica):}

Neem extracts are used to treat various skin diseases,as an antiseptic substance,against endo and ectoparasites or simply as a herbal mouthwash.It is widely used as a non toxic insecticide and pesticide.Recent studies have shown Neem to have significant effects on E.coli,Streptococcus and salmonella.Also neem extracts have been shown to inhibit the growth of streptococcus mutans.Data from these studies suggests that neem stick extracts can reduce the ability of some streptococci to colonize tooth surfaces.

\subsection{Propolis(bees wax):}

Added to toothpaste,it prevents periodontal disease,and is anti-plaque and anti-inflammatory.A brazilian study suggests that,the use of a propolis based mouthwash post oral surgery,aided the repair of surgical wounds and had analgesic and anti-inflammatory effects.

\subsection{Green tea(Camellia sinensis):}

It can be used as a mouthwash or gargle in the treatment of halitosis,laryngitis, sore throat,plaque buildup,tonsillitis, dental caries.It can be used a new,safe method for the treatment of oral diseases in pregnant women and children as it is free of side effects that chemical mouthwashes cause.

\subsection{Cranberry(Vaccinium macrocarpon) juice:}

A study published in the Journal of the American Dental Association reports that a high molecular weight,non-dialysable material(NDM), has the ability to reverse and inhibit the co-aggregation of certain oral bacteria responsible for dental plaque and periodontal disease,

\subsection{Grapefruit(Citrus paradisi):}

Grapefruit extract has a high anti-oxidant content which is useful in annulling bacteria of the oral cavity.

\subsection{0il pulling therapy:}

This is a traditional indian folk remedy that involves swishing oil in the mouth for oral benefits.one tablespoon of oil is swished in the mouth,on an empty stomach,for 15-20 minutes, first thing in the morning,then it is spat out.

\section{Conclusion:}

By this review,it is evident that Tulsi(Ocimum sanctum) is a medicinal plant of great importance, due to its varied application in medicine.For example, as a mouthwash against periodontitis,to improve immunity, as an adjunct to diabetic therapy,as an anti-inflammatory agent,as a hepato-protective agent.

Also,the uses of other similar medicinal plants have been shown,like neem,Green tea,Cranberry,Grapefruit.Among these plants, it is evident that Tulsi has the greatest uses in medicine, and further clinical studies should be performed to study in detail,what our ancestors once knew well,that Tulsi is a plant with many uses in medicine and in this it is truly matchless.

\section{References:}

[1]. Ahmed M, Ahamed RN, Aladakatti RH, Ghosesawar MG. Reversible anti-fertility effect of benzene extract of Ocimum sanctum leaves on sperm parameters and fructose content in rats. J Basic Clin Physiol Pharmacol. 2002; 13(1):51-9.

[2] Amrani S, Harnafi H, Bouanani Nel H, Aziz M, Caid HS, Manfredini S, Besco E, Napolitano M, Bravo E. Hypolipidaemic activity of aqueous Ocimum basilicum extract in acute hyperlipidaemia induced by triton WR-1339 in rats and its antioxidant property. Phytother Res. 2006 ;20(12):1040-5.

[3] Banerjee, S, Parashar R, Kumar A, Rao A R. Modulatory influence of alcoholic extract of Ocimum leaves on carcinogenmetabolizing enzyme activities and reduced glutathione levels in mouse. Nutr Cancer 1996, 25(2): 205-217

[4] Bansod S and Rai M. Antifungal Activity of Essential Oils from Indian Medicinal Plants against Human Pathogenic Aspergillus fumigatus and A. niger. World Journal of Medical Sciences 2008, 3(2): 81-88

[5] Bhargava K P, Singh N. Antistress activity of Ocimum sanctum Linn. Ind J Med Res 1981,73:443-451

[6] Chattopadhyay RR. Hypoglycemic effect of O sanctum leaf extract in normal and streptozotocin diabetic rats. Indian Journal of Experimental Biology 1993,31 891-893

[7] Chiang L C, Ng L T, Cheng P W, Chiang W \& Lin C. Antiviral activities of extracts and selected pure constituents of Ocimum 
[8] Dhar M L, Dhar M M, Dhawan BN, Mehrotra BN \& Roy C.Screening of Indian plants for biological activity, Part I. Indian Journal of Experimental Biology 1968,6 232-247

[9] Source: Periodontal disease | University of Maryland Medical Center http://umm.edu/health/medical/reports/articles/periodontaldisease\#ixzz2rbhYSdU3 University of Maryland Medical Center

[10] 2004 May-Jun;38(3):204-11. Dental plaque as a microbial biofilm. Marsh PD.

[11] 2012 Oct;32(2):69-75. doi: 10.4103/0257-7941.118531.Estimation of salivary and tongue coating pH on chewing household herbal leaves: A randomized controlled trial.Ramesh G, Nagarajappa R, Madhusudan AS, Sandesh N, Batra M, Sharma A, Patel SA.

[12]. fdm.mu-sofia.bg/files/.../4.../12_Oral_Microbial_Ecology_-_english.ppt...

[13] http://en.wikipedia.org/wiki/Halitosis

[14] http://umm.edu/health/medical/reports/articles/periodontal-disease

[15] Cytokine" in John Lackie. A Dictionary of Biomedicine. Oxford University Press. 2010. ISBN 9780199549351

[16] Boggess KA; Society for Maternal-Fetal Medicine Publications Committee. Maternal oral health in pregnancy. Obstet Gynecol. 2008 Apr;111(4):976-86.

[17] .Nakamura CV,Ueda-Nakamura T,Bando E,Melo AF,Cortez DA,Diaz Filho Bp.Antimicrobial activity of Ocimum gratissium L.essential oil.Mem Inst Oswaldo Cruz 1999;94:675-8

[18] Geeta,Vasudevan DM,Kedlaya R,Deepa S,Ballal M.Activity of ocimum sanctum against the enteric pathogens.Indian J Med Sci 2001;55;434-72

[19] Singh S,Majumdar DK,Rehan HMS.Evaluation of anti-inflammatory potential of fixed oil of Ocimum sanctum(holy basil) and its possible mechanism of action.L Ethanopharmacol 1996;54:19-26.

[20] Guth PH,Paulsen G,Nagata H.Histolocic and microcirculatory changes in alchohol induced gastric lesions in the rat:effect of prostaglandin cytoprotection.Gastroenterology 1984;87:1083-90.

[21]. Anderson JW.The role of Dietary carbohydrate and fiber in control of diabetes.Adv Intern Med 1980;26:67-96

[22]. Chattopadhyay RR,sarkar SK,Ganguly S,Medda C,Basu TK.Hepatoprotective activity of Ocimum sanctum leaf extract against paracetamol induced hepatic damage in rats.Indian J Pharmacol 1992;24:163-5

[23] Shklar G,Schwartz JL.Vitamin E inhibits experimental carcinogenesis and tumour angiogenesis.Oral oncol Eur J Cancer 1996;23:114-9 